\title{
Towards a Definition of Symbiotic Relations Between Humans and Machines
}

\author{
Luciano Gamberini ${ }^{(\varpi)}$ and Anna Spagnolli \\ Department of General Psychology \& Human Inspired Technologies Research Centre, \\ Padua University, Padua, Italy \\ \{luciano.gamberini, anna.spagnolli\}@unipd.it
}

\begin{abstract}
What are symbiotic systems? In this short paper, the workshop chairs describe the focus of this workshop and elaborate on the definition of symbiotic human-machine relation.
\end{abstract}

Keywords: Symbiotic system $\cdot$ Human-computer interaction

Symbiotic relationships are probably most known from biology. In a symbiotic relationship between animals and/or plants, some organism closely interacts with another type of organism to ensure its own survival, so that both end up sharing the same habitat [3]. This biological phenomenon, exemplifying a very tight kind of relationship, is used metaphorically ${ }^{1}$ to characterize a growing part of the current human-machine relations, where machines collect users' data that are not even available to the human conscious cognition (e.g. brain signals, social data collected on the web), and then elaborate solutions and make decisions based on such data. Here machines are so interconnected to humans that the term interaction sounds like an understatement [13].

After the pioneering discussion on men-computer [sic] symbiosis stimulated in the early 60 by J.C.R. Licklider [9], the reference to symbiotic human-machine interaction appeared again around 2013 in a European Union ICT workprogram stressing that concept ${ }^{2}$ and has grown ever since, as is partly documented in the Symbiotic Interaction workshops and proceedings $[2,5]$. Surely, in the field of human-computer interaction as well as in philosophy and sociology of technology, the close connection between humans and machines has been emphasized several times: by the concepts of artifacts and embodied cognition, where the propriety of a technology depends on the users' needs and the users' ability depends in turn on the tools s/he is endowed with (e.g., [7]); by the notion of sociotechnical systems, where a technology inevitably includes some symbolic, politic elements as part of the package (e.g., [8]); by the idea of humans as hybrids or cyborgs, which finds real-life incarnations in medical prosthesis and augmentation devices (e.g., [1]). However, the term symbiotic emphasizes a noteworthy change

${ }^{1}$ Of course, a metaphor is able to illuminate some aspects of a domain by similarity with some other domain [12], but not all aspects. Where the biological metaphor stops is in referring to two leaving organisms in partnership, since a symbiotic technology is not credited to share the same needs (and rights) as the human being to which it is joined.

2 http://cordis.europa.eu/fp7/ict/docs/ict-wp2013-10-7-2013-with-cover-issn.pdf. 
in the interdependence between humans and machines, pointing to an intimate kind of relation that is made possible by the synergic advances in physiological computing, biometrics, sensing technologies, and machine learning, often combined with the ubiquity of networked devices. Users do not necessarily need to be aware of what is happening while machines help themselves with information, since this process mostly occurs implicitly [6], relying on human biosignals that are out of grasp to the individual user (e.g., [10]) or on billions of traces left by users of networked devices. A symbiotic device does not need to be reprogrammed or fine-tuned: on the contrary, it can learn, sense the context, evolve in close relation with the environment by analyzing the consequences of processes jointly started with humans.

So, although very different in purpose and tradition, all the "symbiotic advances" converge in technological products that tap directly the user to get input information. and evolve their own intelligence on the basis of their performance. The pervasiveness, ubiquity and consequentiality of such devices in everyday life justify their being singled out, monitored and reflected upon. The adoption of a dedicated label for such devices, such as 'symbiotic', is meant to encourage such process.

Elaborating on the description of symbiotic interaction first proposed in 2014 [4], we can identify symbiotic systems as those computerized devices that can autonomously fetch information from the users, interpret such information to model the user and then make decisions with the ultimate goal of simplifying the users' task, clarifying possible issues, or refining a service for them (e.g., recommendations); and finally to evolve and become more efficient by learning from previous interaction. To represent this process in terms of human-machine interaction, we can extend Norman's model of action [11] into something similar to what is shown in Fig. 1.

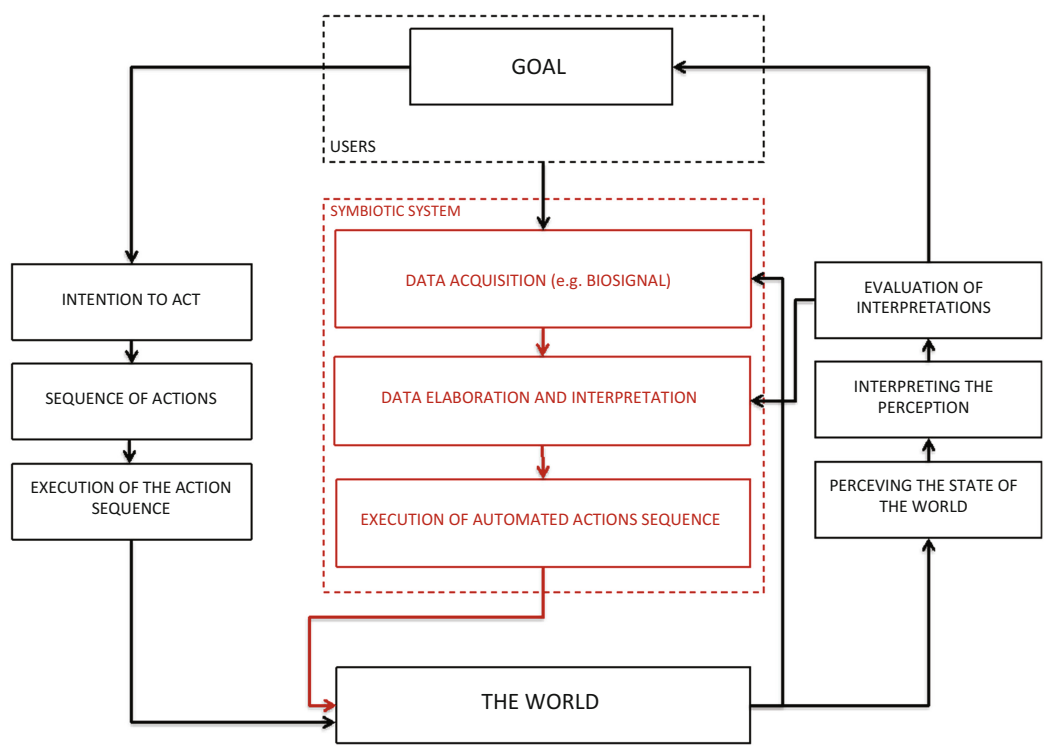

Fig. 1. A modification of Donald Normans action model to include a symbiotic system. 
Since this process is meant to release the user from the burden of providing the information necessary to enjoy some improved, tailored service, it is in continuity with HCI principle of usability and intuitiveness. However, this process works so far in the background to become elusive and so peculiar in its interpretation of the users' goals to misjudge them. There is room for reflection then on the risks for users, let alone the clear advantages brought about by symbiotic machines. Advantages will be described by almost all chapters in this book, each one illustrating some applications of symbiotic technology; risks will be highlighted and reflected upon in the next chapter.

Acknowledgments. The present reflections benefit from the work carried out within the EU project MindSee (grant agreement n. 611570).

\section{References}

1. Barfield, W.: Cyber-Humans: Our Future with Machines. Springer, Cham (2015)

2. Blankertz, B., Jacucci, G., Gamberini, L., Spagnolli, A., Freeman, J. (eds.): Symbiotic 2015. LNCS, vol. 9359. Springer, Cham (2015). doi:10.1007/978-3-319-24917-9

3. Douglas, A.E.: The symbiotic habit. Princeton University Press (2010)

4. Jacucci, G., Spagnolli, A., Freeman, J., Gamberini, L.: Symbiotic interaction: a critical definition and comparison to other human-computer paradigms. In: Jacucci, G., Gamberini, L., Freeman, J., Spagnolli, A. (eds.) Symbiotic 2014. LNCS, vol. 8820, pp. 3-20. Springer, Cham (2014). doi:10.1007/978-3-319-13500-7_1

5. Jacucci, G., Gamberini, L., Freeman, J., Spagnolli, A. (eds.): Symbiotic 2014. LNCS, vol. 8820. Springer, Cham (2014). doi:10.1007/978-3-319-13500-7

6. Janlert, L.E., Stolterman, E.: The Meaning of Interactivity-Some Proposals for Definitions and Measures. Human-Computer Interaction, pp. 1-36 (2016)

7. Kirsh, D.: Embodied cognition and the magical future of interaction design. ACM Trans. Comput.-Hum. Interact. (TOCHI) 20(1), 3 (2013)

8. Kling, R.: Social analyses of computing: theoretical perspectives in recent empirical research. ACM Comput. Surv. (CSUR) 12(1), 61-110 (1980)

9. Licklider, J.C.: Man-computer symbiosis. IRE Trans. Hum. Factors Electron. 1, 4-11 (1960)

10. Negri, P., Gamberini, L., Cutini, S.: A review of the research on subliminal techniques for implicit interaction in symbiotic systems. In: Jacucci, G., Gamberini, L., Freeman, J., Spagnolli, A. (eds.) Symbiotic 2014. LNCS, vol. 8820, pp. 47-58. Springer, Cham (2014). doi:10.1007/978-3-319-13500-7_4

11. Norman, D.A.: The Design of Everyday Things. Basic Book, New York (1988)

12. Ortony, A.: Metaphor and Thought. Cambridge University Press, Cambridge (1993)

13. Verbeek, P.P.: Beyond interaction: a short introduction to mediation theory. Interactions 22(3), 26-31 (2015) 
Open Access This chapter is licensed under the terms of the Creative Commons Attribution 4.0 International License (http://creativecommons.org/licenses/by/4.0/), which permits use, sharing, adaptation, distribution and reproduction in any medium or format, as long as you give appropriate credit to the original author(s) and the source, provide a link to the Creative Commons license and indicate if changes were made.

The images or other third party material in this chapter are included in the chapter's Creative Commons license, unless indicated otherwise in a credit line to the material. If material is not included in the chapter's Creative Commons license and your intended use is not permitted by statutory regulation or exceeds the permitted use, you will need to obtain permission directly from the copyright holder.

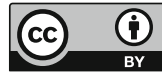

